\title{
Intravitreal chemotherapy for the treatment of recurrent intraocular lymphoma
}

\author{
Marc D de Smet, Virginia Stark Vancs, David Kohler, Diane Solomon, Chi Chao Chan
}

Clinical Immunology Section, Laboratory of Immunology, National Eye Institute,

Netherlands

M D de Smet

Immunopathology Section, Laboratory of Immunology, National

Eye Institute,

Netherlands

C C Chan

Division of Cancer Treatment, National Cancer Institute, Netherlands

V S Vancs

Pharmacy

Department, Warren

G Magnuson Clinical

Center, Netherlands

D Kohler

Cytopathology Section, Laboratory of

Pathology, National

Cancer Institute,

National Institutes of

Health, Netherlands

D Solomon

Department of

Ophthalmology,

Academic Medical

Center, University of

Amsterdam,

Netherlands

M D de Smet

Correspondence to: Marc D de Smet, $\mathrm{MD}$ Academic Medical Center, University of Amsterdam, Kamer G2-217,

Meibergdreef 9, Amsterdam 1105 AZ, Netherlands.

Accepted for publication 23 October 1998

\begin{abstract}
Aim-To develop and assess a protocol for the treatment of intraocular lymphoma by intravitreal injection of methotrexate and thiotepa.

Methods-A patient with intraocular nonHodgkin's lymphoma which recurred after radiotherapy and repeated systemic chemotherapeutic regimens underwent repeated intravitreal injections of methotrexate and thiotepa. The patient was closely monitored by cytology, anterior chamber flare measurements, IL-10 and IL-6 levels. Methotrexate drug clearance studies were performed on vitreous samples taken before each injection.

Results-Complete tumour clearance was achieved by the third week of therapy. IL-10 and IL-6 levels quickly dropped to barely detectable levels as the tumour was cleared from the eye. Flare measurements decreased from 500 to 15 photons/s over the same time. A plot of the methotrexate levels over time revealed a first order kinetic rate of elimination with an effective tumoricidal intravitreal dose persisting for $\mathbf{5}$ days after injection.

Conclusion-Intravitreal chemotherapy for the treatment of recurrent intraocular lymphoma appears effective in prolonging local remission of ocular disease even in the presence of an aggressively growing tumour. A single intravitreal injection of methotrexate can lead to a prolonged tumoricidal concentration lasting for a longer period than that achieved by systemic administration.

(Br F Ophthalmol 1999;83:448-451)
\end{abstract}

Primary central nervous system (CNS) and intraocular non-Hodgkin's lymphoma (NHL) is initially treated with systemic chemotherapy administered alone or in combination with radiotherapy. ${ }^{1-5}$ For disease limited to the eye, radiotherapy alone may lead to a prolonged disease free state. ${ }^{36}$ However, local or CNS recurrence is common. A relapse free state at 5 years is less than $33 \% .{ }^{78}$ In addition, radiation to the eye can have significant toxicity including radiation retinopathy, ${ }^{9-11}$ optic neuropathy, ${ }^{12}{ }^{13}$ dry eye syndrome, and prolonged corneal epithelial defects. ${ }^{31415}$ While surface complications can be minimised by adjusting the radiation portals, the risks of posterior complications increase with radiation dosage, the presence of concomitant chemotherapy, age, and ocular vascular impairment. ${ }^{1016}$
To date, the treatment of ocular recurrences has received little attention. Additional radiotherapy is possible when the maximum allowed radiation dose has not been reached, but this form of therapy is often no longer available without considerable risk. Systemic multiagent chemotherapy can be considered. ${ }^{17}$ However, systemic toxicity can be quite significant, ${ }^{18}$ and is often insufficient to clear the ocular tumour. Another approach is direct intraocular injection. ${ }^{19} 20$ The latter has the advantage of delivering a substantial local dose without significantly increasing systemic toxicity. This article reports on the effect of direct intraocular injection of combined methotrexate and thiotepa into an eye with a recurrent NHL, which had persistent disease despite receiving 45 Gy of radiotherapy and systemic multiagent chemotherapy.

\section{Case report}

A 50 year old white woman was diagnosed with a large cell lymphoma involving the brain and both eyes. The diagnosis was confirmed by performing a vitrectomy on her left eye. She was treated with a combination of $45 \mathrm{~Gy}$ of radiation to the eyes and brain, and intrathecal cytarabine (Ara-C). Over the next 2 years, she developed significant cataracts which required surgery with intraocular lens implantation. She also developed chronic open angle glaucoma in her left eye. Four years after the initial diagnosis, she complained of increased floaters and blurred vision in her left eye. She was noted to have significant vitreous haze precluding visualisation of her posterior pole. Her visual acuity was light perception in the involved eye. Four months later, lymphomatous cells were noted in her cerebrospinal fluid.

She was started on an investigational regimen of systemic and intrathecal chemotherapy approved by the institutional review board of the National Cancer Institute. The intravenous regimen included methotrexate $\left(1.5 \mathrm{~g} / \mathrm{m}^{2}\right.$ bolus over 1 hour followed by $300 \mathrm{mg} / \mathrm{m}^{2}$ per hour over 23 hours for a total of $8.4 \mathrm{~g}$ over a 24 hour period), thiotepa (35 mg/m $\mathrm{m}^{2}$ on day 1 ), vincristine $\left(1.4 \mathrm{mg} / \mathrm{m}^{2}\right)$, and Decadron (dexamethasone, $6 \mathrm{mg}$ every 6 hours $\times 20$ doses). Intrathecal chemotherapy included Ara-C (50 mg on days 6, 13, and 19 of each cycle), and methotrexate (12 $\mathrm{mg}$ on days 9 and 16 of each cycle) (Protocol NCI no T94-0054). No tumour cells were detected in the CSF after the second cycle of chemotherapy. As per protocol, she was given a further two cycles after achieving clinical remission in her CSF. In her involved eye the vitreous haze decreased with each cycle of systemic chemotherapy. How- 
ever, at the beginning of each subsequent cycle, there was an increase in haze. By the second cycle, a hypopyon would transiently form at the onset of each cycle.

After four cycles of chemotherapy, as per protocol, the patient received no further systemic or intrathecal treatment. Further ocular radiotherapy was not deemed appropriate in this patient since she had already received maximum doses of radiation to both eyes. She did not want to have her eye enucleated, despite having only light perception vision. The patient agreed to a trial of local intravitreal therapy, since partial resolution of the tumour had been observed with each cycle of systemic chemotherapy.

Approval was obtained from the investigational review board of the National Cancer Institute. A regimen consisting of both thiotepa and methotrexate was chosen. At each injection, she was monitored for tumour response by cytology as well as by a number of surrogate markers including cell/flare photometry, IL-10 and IL-6 levels. Initially methotrexate was given twice weekly, while thiotepa was given once a week for 3 weeks. Once her cytology became negative, she was given biweekly injections $\times 3$, monthly $\times 3$, and followed prospectively with no further intervention. Her vitreous volume by ultrasound was $4.07 \mathrm{ml}$. To achieve a peak intravitreal methotrexate concentration of 100 $\mu \mathrm{g} / \mathrm{ml}$, each injection of $0.1 \mathrm{ml}$ contained 4 $\mathrm{mg} / \mathrm{ml}$ of methotrexate. Thiotepa was given at a concentration of $2 \mathrm{mg} / 0.1 \mathrm{ml}$.

Before each injection, an appropriate volume of vitreous fluid was removed to leave the eye slightly hypotonic at the end of the procedure. This sample was used for the analyses mentioned below. Methotrexate injections were well tolerated and did not cause any discomfort. Thiotepa was associated with a significant pressure rise about $2-4$ hours after injection. This was observed with the first three injections. On one occasion, the pressure rose to 60 $\mathrm{mm} \mathrm{Hg}$, but normalisation was achieved with an anterior chamber paracentesis. Subsequent to this event, the visual acuity dropped to bare LP/NLP. A mild vitreous haemorrhage developed after the second injection which cleared slowly. On cytology, complete clearance of all tumour in the vitreous sample was achieved during the third week of therapy. A pars plana vitrectomy, performed at the end of the third week, confirmed that no viable tumour cells were present in the vitreous cavity or in the peripheral vitreous skirt. At surgery, a pale excavated optic nerve head with a cup/disc ratio of 1.0 was noted. The patient completed the course of intravitreal chemotherapy without difficulty. Eighteen months later (30 months after the initiation of combined therapy), she had no evidence of a recurrence.

\section{Methods}

Flare measurements were performed using a standard procedure established for the use of the Kowa laser cell flare meter FC1000.$^{21}{ }^{22}$ In essence, seven consecutive measurements with a difference in background (BG1 versus BG2) of less than $15 \%$ were taken. The highest and lowest values were eliminated before averaging the remaining values.

Vitreous sampling and intravitreal injections were given $3 \mathrm{~mm}$ posterior to the limbus following retrobulbar anaesthesia and complete ocular akinesia. After ocular disinfection with a $10 \%$ betadine solution, $0.3 \mathrm{ml}$ of vitreous fluid was removed form the eye. The tuberculin syringe was separated from the 30 gauge needle inserted in the vitreous cavity. It was replaced by a syringe containing a single chemotherapeutic agent (methotrexate $4 \mathrm{mg}$ / $\mathrm{ml}$; thiotepa $20 \mathrm{mg} / \mathrm{ml}$ ). A total of $0.1 \mathrm{ml}$ was injected over a 5 minute period. If a second agent needed to be given, the following syringe was substituted, and the injection given over 5 minutes. At the end of the procedure the eye was left somewhat hypotonous, and gentamicin ointment was placed in the conjunctival sac. In order to determine the maximal intravitreal methotrexate dose, on two occasions a vitreous sample was taken 5 minutes after completing the injection.

The vitreous sample was carefully partitioned in three fractions. The first was sent to cytopathology, where the material was immediately placed on sialinated slides, air dried, and stained to identify tumour cells. ${ }^{23}$ The other two fractions were immediately frozen at $-70^{\circ} \mathrm{C}$ to allow subsequent batch determinations of cytokine and methotrexate levels. Interleukin 6 and 10 were determined using a standard enzyme linked immunoassay (ELISA kit, Endogen, Cambridge, MA, USA). Methotrexate levels were determined using a fluorescence polarisation immunoassay (TDx/TDxFLx, Abbott Laboratories, Abbott Park, IL, USA).

\section{Results}

Our primary endpoint was cytological clearance of the tumour. As indicated in the case report, this was achieved by the third week of therapy. In addition, several surrogate markers were followed. Flare photometry was a sensitive indicator of the severity of blood ocular barrier breakdown. Initially, measurements were above 550 photons $/ \mathrm{ms}$. These levels rapidly dropped as the tumour was cleared from the eye and integrity of the barrier was rapidly re-established. By 21 days, the level had dropped to below 50 photons/ms, where it remained thereafter. Initially raised IL-10 levels quickly fell as the tumour was eradicated from the eye (Table 1). The increase noted on day 85 was associated with an even higher level of IL-6. This has previously been reported as indicative of intraocular inflammation, and not of a recurrence of lymphoma. ${ }^{24}$ Cytological

Table 1 Intravitreal IL-6 and IL-10 levels at various times since onset of intravitreal therapy

\begin{tabular}{lcc}
\hline Day of sampling & $I L-6(\mathrm{pg} / \mathrm{ml})$ & $I L-10(\mathrm{pg} / \mathrm{ml})$ \\
\hline 0 & 186 & 5100 \\
11 & 250 & 200 \\
24 & 50 & 0 \\
87 & 2586 & 1856 \\
112 & 468 & 0 \\
156 & 0 & 0 \\
\hline
\end{tabular}




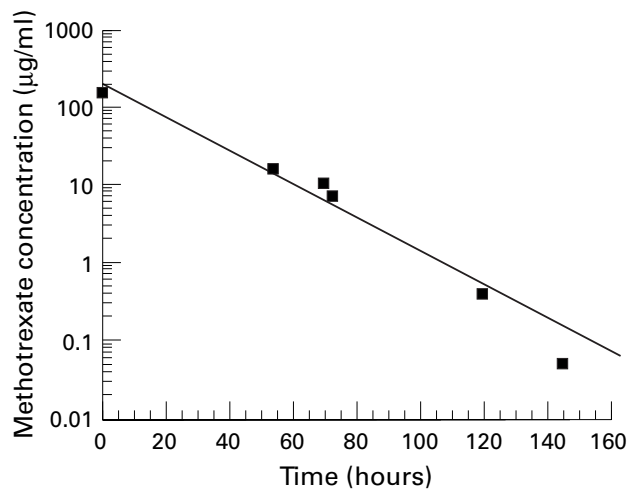

Figure 1 Intravitreal concentrations at various time points following intravitreal injection of methotrexate $(400 \mu \mathrm{g})$.

Each time point corresponds to a different baseline injection, as each sample was taken before the subsequent injection of methotrexate.

examination at that time did not show the presence of any intraocular tumour.

Since vitreous sampling occurred before the administration of chemotherapy and was performed at different time points after the injection of the previous dose, it was possible to roughly plot drug levels at various time points after methotrexate injection (Fig 1). On a semilogarithmic plot, a linear relation is observed, suggesting a first order kinetic rate of elimination. Given that a dosage above $1 \mu \mathrm{mol} / 1$ is considered tumoricidal, it suggests that a single intravitreous dose remains effective for about 5 days.

\section{Discussion}

This patient with persistent intraocular lymphoma following systemic and intrathecal high dose therapy refused to have her eye enucleated. In an attempt to allow her to keep her eye, and to eradicate the lymphoma, we opted for intravitreal therapy. Radiotherapy had been ruled out as an option, as she had previously received maximal therapy with $45 \mathrm{~Gy}$. Although it is sometimes possible to deliver even more radiation, she had developed a dry eye syndrome following her previous radiation treatment and occasionally suffered from corneal erosions.

With each systemic methotrexate cycle, she had shown a significant decrease in vitreal haze, but this improvement was only transient. We speculated that the tumour recurred because the intraocular methotrexate levels could not be sustained for long enough above a minimal tumoricidal concentration. Current CNS lymphoma protocols combine systemic and intrathecal therapy in order to boost drug delivery. Direct administration of chemotherapy to the CSF bypasses the blood-brain barrier and delivers a high sustained dose of chemotherapy to the meninges. ${ }^{2}{ }^{18}{ }^{25}$ Using this approach, the minimal methotrexate tumoricidal concentration is maintained within the CSF for a minimum of 48 hours, compared with a few hours when given systemically. Methotrexate given intrathecally is very effective at preventing leptomeningeal recurrences. The bolus injected within the CSF equilibrates at about $100 \mu \mathrm{g} / \mathrm{ml}$. Based on ultrasonographic measurements of the vitreous volume, the intravitreal methotrexate dose was selected to achieve a similar intraocular concentration. Since the ocular disease was highly aggressive and recurrent, we were not sure that methotrexate alone would be able to eradicate the intraocular lymphoma. Thus, multiagent rather than single agent therapy was considered more appropriate. Thiotepa is the only other drug which so far has been administered intravitreally to treat a malignancy. ${ }^{19}$

The intravitreal dosing regimen was based on available data from intravenous and intrathecal treatment. ${ }^{26}{ }^{27}$ Intrathecally, methotrexate levels are maintained at tumoricidal levels for 48 hours. Thus, biweekly injections were considered appropriate, since ocular clearance is slower than from the CSF. ${ }^{18}$ For thiotepa, available evidence suggested that administration once a week would be sufficient. High frequency dosing was maintained until tumour clearance was achieved. The follow up regimen was chosen to ensure consolidation of remission. ${ }^{28}{ }^{29}$ The decision to move on to the consolidation regimen was based on cytological confirmation of tumour clearance.

Surrogate markers supported the results obtained from cytology. Blood-ocular barrier integrity returned to virtually normal levels once the tumour was eradicated from the eye. Intravitreal IL-10 levels, a cytokine secreted by lymphoma cells, paralleled the intraocular tumour burden as previously suggested by Chan and coworkers. ${ }^{24}{ }^{30-32}$ It was absent at the time the two last doses were administered.

The visual acuity drop noted in this patient was temporally related to an acute rise in intraocular pressure. This patient was known to have pre-existing open angle glaucoma Increased pressure was only observed with thiotepa. Its cause is unknown. Whether thiotepa is necessary in the treatment of recurrent intraocular lymphoma can be debated. However, single agent treatment in the setting of recurrent tumour is not usually recommended. Another potential choice is direct intraocular treatment with dexamethasone or a long acting steroid preparation.

In this patient, intravitreal chemotherapy was successful in eradicating an aggressive lymphoma, and leading to prolonged remission. It should be noted that the treatment was given in conjunction with aggressive systemic therapy. This is in agreement with the approach used by Fishburne et al in a similar group of patients. ${ }^{20}$ Since this tumour has a propensity to disseminate to the other eye and the brain, it is important to consider local ocular management as one of the required components in the management of this tumour. In addition to local treatment, aggressive systemic and intrathecal chemotherapy are essential to ensure a prolonged remission. It is hoped that this approach may provide prolonged survival while maintaining good levels of visual function.

Presented in part at the American Academy of Ophthalmology Annual Meeting, Atlanta, November 1995. 
1 DeAngelis LM, Yahalom JY, Heinemann M-H, et al. Primary CNS lymphoma: combined treatment with
chemotherapy and radiotherapy. Neurology 1990;40:80-6.

2 DeAngelis LM, Yahalom J, Thaler HT, et al. Combined modality therapy for primary CNS lymphoma. $\mathcal{F}$ Clin Onco 1992;10:635-43.

3 Char DH, Ljung BM, Miller T, et al. Primary intraocular lymphoma (ocular reticulum cell sarcoma) diagnosis and management. Ophthalmology 1988;95:625-30.

4 Boiardi A, Silvani A, Valentini S, et al. Chemotherapy as first treatment for primary malignant non-Hodgkin's lymphoma of the central nervous system preliminary data. f Neurol 1993;241:96-100.

5 Chamberlain MC, Levin VA. Adjuvant chemotherapy for primary lymphoma of the central nervous system. Arch Neurol 1990;47:1113-16.

6 Pollack IF, Lunsford LD, Flickinger JC, et al. Prognostic factors in the diagnosis and treatment of primary central factors in the diagnosis and treatment of primary cent

7 Rouwen AJP, Wijermans PW, Boen-Tan TN, et al. Intraocular non-Hodgkin's lymphoma treated with systemic and intrathecal chemotherapy and radiotherapy. A case report intrathecal chemotherapy and radiotherapy. A case report and review of the lite

8 Peterson K, Gordon KB, Heinemann MH, et al. The clinical spectrum of ocular lymphoma. Cancer 1993;72:843-9.

9 Brown GC, Shields JA, Sanborn G, et al. Radiation retinopathy. Ophthalmology 1982;89:1494-501.

10 Archer DB, Amoaku WMK, Gardiner TA. Radiation retinopathy-clinical, histopathological, ultrastructural and experimental correlations. Eye 1991;5:239-51.

11 Chee PHY. Radiation retinopathy. $A m$ f Ophthalmol 1968;66:860-5

12 De la Paz MA, Boniuk M. Fundus manifestations of orbital disease and treatment of orbital disease. Surv Ophthalmol 1995;40:3-21.

13 Brown GC, Shields JA, Sanborn G, et al. Radiation optic neuropathy. Ophthalmology 1982;89:1489-93.

14 Chan RC, Shukovsky LJ. Effects of irradiation on the eye. Ther Radiol 1976;120:673-5.

15 Merriam GR, Jr, Szechter A, Focht EF. The effects of ionizing radiations on the eye. Front Radiation Ther Onc 1972;6: 346-85.

16 Viebahn M, Barricks ME, Osterloh MD. Synergism between diabetic and radiation retinopathy: case report and review. Br f Ophthalmol 1991;75:629-62.

17 Plowman PN, Montefiore DS, Lightman S. Multiagent chemotherapy in the salvage cure of ocular lymphom relapsing after radiotherapy. Clin Oncol 1993;5:315-16. 18 de Smet MD, Stark-Vancs V, Kohler DR, et al. Intraocular
levels of methotrexate after intravenous administration. $A m$ F Ophthalmol 1996;121: 442-4.
19 Seregard S, Kock E, af Trampe E. Intravitreal chemotherapy for recurrent retinoblastoma in an only eye. $\mathrm{Br} \mathcal{F}$ Ophthalmol 1995;79:194-5.

20 Fisbburne BC, Wilson DJ, Rosenbaum JT, et al. Intravitreal methotrexate as an adjunctive treatment of intraocular lymphoma. Arch Ophthalmol 1997;115:1152-6.

21 Nussenblatt RB, de Smet M, Podgor M, et al. The use of the flare photometry in the detection of cytomegalic virus retinitis in AIDS patients. AIDS 1994;8:135-6.

22 Guex-Crosier Y, Pittet N, Herbort CP. Evaluation of laser flare-cell photometry in the appraisal and management of intraocular inflammation in uveitis. Ophthalmology 1994; 101:728-35.

23 Davis JL, Solomon D, Nussenblatt RB, et al. Immunocytochemical staining of vitreous cells. Indications, techniques, and results. Ophthalmology 1992;99:250-6.

24 Chan CC, Whitcup SM, Solomon D, et al. Interleukin-10 in the vitreous of patients with primary intraocular lymphoma. Am f Ophthalmol 1995;120:671-3.

25 Borsi JD, Moe PI A comparative study on the pharmacokinetics of methotrexate in a dose range of $0.5 \mathrm{~g}$ to $33.6 \mathrm{~g} / \mathrm{m}^{2}$ in children with acute lymphoblastic leukemia. Cancer 1987;60:5-13.

26 Ettinger LJ, Chervinsky DS, Freeman AI, et al. Pharmacokinetics of methotrexate following intravenous and intraventricular administration in acute lymphocytic leukemia and non Hodgkin's lymphoma. Cancer 1982;50:1676-82.

27 Shapiro WR, Young DF, Mehta BM. Methotrexate: distribution in cerebrospinal fluid after intravenous, ventricular and lumbar injections. N Engl f Med 1975;293: ventricular $161-6$.

28 Baumann MA, Ritch PS, Hande KR, et al. Treatment of intraocular lymphoma with high-dose Ara-C. Cancer 1986; 57:1273-5.

29 Liang B, Grant R, Junck L, et al. Primary central nervous system lymphoma: treatment with multiagent systemic and intrathecal chemotherapy with radiation therapy. Int $\mathcal{F}$ Oncol 1993;3:1001-4.

30 Rousset F, Garcia E, Defrance T, et al. Interleukin-10 is a potent growth and differentiation factor for activated human B lymphocytes. Proc Nat Acad Sci USA 1992;89. 1890-3.

31 Blay JY, Burdin N, Rousset F, et al. Serum interleukin-10 in non-Hodgkin's lymphoma: a prognostic factor. Blood 1993;82:2169-74

32 Ford RJ, Tamayo A, Ambrus JL. The role of growth factors in human lymphomas. Curr Top Microbiol Immunol 1992;182:341-7. 